\title{
REMARQUES SUR RELIGION ET POLITIQUE CHEZ LÉVINAS
}

Fabio Ciaramelli

SÍNTESE - O Autor investiga os conceitos de politica e religiäo na obra do filósofo Emmanuel Lévinas, destacando principalmente suas mútuas implicações e detendo-se na característica corretiva da concepção levisiana de religiảo com relação à política que, deixada a ela mesma em um processo de autojustificação, degenera em totalidade.

ABSTRACT - The author investigates the concepts of "politics" and "religion" in the writings of the philosopher Emmanuel Lévinas; he, mainly, emphasizes their related implications, and examines the "corrective" characteristic of Lévina's conception of "religion" in relation to "politics", when politics it is left to a process of self-justification it results in totality.

"La politique - écrit Lévinas dans Totalité et Infini - tend à la reconnaissance réciproque, c'est-à-dire à l'égalité; elle assure le bonheur. Et la loi politique achève et consacre la lutte pour la reconnaissance. La religion est Désir et non point lutte pour la reconnaissance. Elle est le "surplus possible dans une société d'égaux", celui de la glorieuse humilité, de la responsabilité et du sacrifice, condition de l'égalité ellemême" (p. 35, souligné par moi).

Nous sommes là immédiatement aux prises avec un paradoxe: ce que deux lignes plus haut Lévinas appelle "la distance qui [...] sépare politique et religion" (ibid.), à y regarder de près, dessine un cercle. Ces deux dimensions empiètent l'une sur l'autre et il faut les penser ensemble. Si la "religion" est un "surplus possible dans une société d'égaux", il faut qu'elle suppose instaurée l'égalité - une égalité qui n'est pas naturelle, qui ne va pas de soi, mais qui doit être posée par la loi politique où aboutit la lutte pour la reconnaissance; et toutefois, la "religion" comme articulation d'une responsablité irréfutable pour le prochain, ${ }^{1}$ est à la fois condition de cette même égalité sans laquelle elle ne serait pas possible comme surplus. Par conséquent, la religion suppose l'égalité politique, dont toutefois elle est une condition.

Il y a donc ici inhérence réciproque entre politique et religion, et l'on ne saurait déduire l'une de l'autre d'une façon rectiligne. La religion est le surplus possible dans une societé d'égaux dans la mesure où elle est d'abord pensée comme condition de l'égalisation. Je dis bien égalisation, et non simple égalité, pour souligner qu'il s'agit

1 Quant à la référence au sacrifice dans le passage cité au début, il est peut-être éclairant de remarquer qu'au sujet de la traduction de la Bible par Buber, Lévinas écrit ceci: "pour le sacrifice, il rappelle admirablement l'idée de proximité que contient le terme hébraïque de Korban en le traduisant par l'approche" (Hors sujet, p. 25). Voir à ce sujet E. Weber, "Approche, Ritspa, Esther. Quelques remarques sur M. Buber et E. Lévinas", Emmanuel Lévinas, Cahier de l'Herne dirigé par M. Abensour et C. Chalier, 1991, p. 454-463. 
ici du résultat de la lutte pour la reconnaissance, qui établit une dimension symbolique, extérieure à la societé, où les sujets peuvent se reconnaître comme égaux. Or, précisément à l'égard d'un tel ordre politique - à la fois institué et symbolique - la religion, indépendamment de sa signification confessionnelle et théologique, joue un rôle essentiel. C'est sur ce rôle en quelque sorte structural de mise en scène d'une condition et d'une limite du politique que porte l'interrogation philosophique de la religion à laquelle le texte de Lévinas permet de penser. ${ }^{2}$

La question sous-jacente à cette exigence de poser et voir dans la religion une condition de la politique, on peut, me semble-t-il, la décéler dans le passage décisif que voici, tiré des Conclusions de Totalité et Infini: "Dans la mesure où le visage d'Autrui nous met en relation avec le tiers, le rapport métaphisique de Moi à Autrui, se coule dans la forme du Nous, aspire à un État, aux institutions, aux lois qui sont la source de l'universalité. Mais la politique laissée à elle-même, porte en elle une tyrannie. Elle déforme le Moi et l'Autre, qui l'ont suscitée, car elle les juge selon le règles universelles et, par là même, comme par contumace" (p. 276, souligné par moi).

La tyrannie immanente à la politique laissée à elle-même est la tyrannie de la totalité, où la politique s'invertit en dénégation de la reconnaissance réciproque qu'elle cherchait, et devient impossibilité de l'égalité car avant tout elle oblitère la différence. La condition de l'égalité est donc à penser d'abord comme une résistance à la totalisation, à partir d'une distance infranchissable, d'une limite à la réciprocité, dont la figuration est fournie par l'extériorité de la société a l'égard d'elle-même mise en scène par la religion.

Il faut ici rappler la différence entre le "sacré" et le religieux au sens de Lévinas. Le sacré comporte l'idée d'une communion fusionnelle, où les différences s'evanouissent au profit de l'unité. L'idée de totalité ressent la fascination du sacré, de l'unité qu'il implique et vers laquelle il tend. Le social pensé à partir de la totalité est hanté par l'unité, et le sacré contribue à l'uniformer. Dans ce sens il y a une solidarité extrême entre les pensées dialectiques du social en termes de totalité, et les pensées mystiques de l'approche du scaré comme fusion et communion: le fantasme de l'unité, la hantise de l'Un orientent les unes et les autres, qui au fond se représentent l'approche de l'Un comme une réalité s'accomplissant dans l'expérience, grâce à l'abolition de toute distance, de toute différence, de toute limite.

Le religieux au sens de Lévinas est lié à l'opposition entre le sacré et le "saint". La sainteté est à la base du religieux: elle s'axe autour de l'idée de séparation, en tant que sauvegarde de la différence, méfiance envers la symétrie et envers l'union fusionnelle. Cela a des retentissements dans la conception de la religion au sens confessionnel, où Lévinas s'oppose à toute forme de mysticisme, ce qui toutefois ne nous retiendra pas ici; mais cela a surtout des retentissements dans la pensée du religieux comme limite et condition du politique.

L'allergie pour toute philosophie de la communion induit Lévinas à s'éloigner radicalement d'une pensée du social à la lumière de sa réalisation fusionnelle, où il perdrait justement sa pluralité constitutive, et se réduirait à l'unité. Au contraire, Lévinas

2 Je me propose ici uniquement d'interroger le sens symbolique de la religion comme condition de l'égalité instaurée para la lutte pour la reconnaissance, et je vais essayer d'en montrer la fécondité pour une critique du politique pensé en termes de totalité. Le discours positif sur la religion comme "surplus possible dans une société d'égaux", donc sur la religion comme témoignage prophétique et kérygme éthique - à quoi Lévinas consacre l'essentiel de ses écrits sur le judaïsme - découle sans doute des mêmes prémisses, mais dépasse les limites de mon propos. 
considère que le "face-à-face" est la situation ultime et la donnée irréductible du social précisément parce que dans le face-à-face la proximité n'abolit pas la distance où peut s'enraciner le désir.

Le "lieu" propre du désir - comme relation à autrui irréductible au besoin, et dès lors réfractaire à la totalité - est donc la pluralité humaine, pensée d'une façon radicale comme "un exister multiple, le pluralisme", c'est-à-dire une "multiplicité radicale", à distinguer foncièrement de la "multiplicité numérique", qui, quant à elle, "reste sans défense contre la totalisation" (p. 195). Par contre, le modèle du social pensé à partir de l'expérience et de l'idée de la totalité, c'est le modèle de la réciprocité et de l'homogénéité, où la relation entre les termes séparés est une relation formelle et interchangeable. Dès lors le social est posé comme un sujet collectif, c'est-à-dire comme une somme de sujets individuels dont les volontés peut-être sont encore discordantes, mais malgré tout promises à un accord rationnel conforme à la leur nature profonde, que la théorie a précisément por tâche de dévoiler. A quoi Lévinas oppose, dès l'article sur "Le Moi et la totalité" de 1954, cette assertion péremptoire: "Nous n'est pas le pluriel de Je" (Entre nous. Essais sur le penser-à-l'autre, p. 49).

L'opposition de Lévinas à une pensée du social à partir de l'expérience de la totalité a ici une double implication. Le social n'est ni la négation ou le dépassement de l'individuel, ni non plus l'élargissement d'une subjectivité isolée et solitaire. La dissolution de l'ipséité dans le collectif n'est qu'une confirmation du monisme ou du solipsisme. Ces deux attitudes reviennent au même, et impliquent une confusion entre le pluralisme de la société et la multiplicité numérique objectivable. C'est dans ce sens que Lévinas refuse d'aborder les rapports humains en sociologue, refuse de les objectiver, et s'interroge sur la relation qu'entretiennt des êtres radicalement différents, pourtant capables de fraternité. Il dit, lors d'un débat à la Société française de philosophie sur Totalité et Infini: "Les hommes sont absolument différents les uns des autres, le concept homme est le seul qui n'ait pas de compréhension, puisque chaque homme est absolument différent de l'autre. Le concept homme a seulement une extension, et c'est la fraternité humaine. Et ce serait tout de même très étrange, si le terme homme désignait un genre: a-t-on jamais vu des individus d'un genre: a-t-on jamais vu des individus d'un genre qui se mettent à fraterniser? " $^{3} \mathrm{D}$ 'où la nécessité d'une interrogation philosophique et phénoménologique des implications des relations humaines. Et c'est dans ce même cadre que Lévinas élucide le sens et les limites de sa référence à la religion. "Mon point de départ est absolument non théologhique": "je ne voudrais rien définir par Dieu, parce que c'est l'humain que je connais. C'est Dieu que je peux définir par les relations humaines et non pas inversement". "Je ne refuse pas le terme de religieux, mais je l'adopte pour désigner la situation où le sujet existe dans l'impossibilité de se cacher. Je ne pars pas de l'existence d'un être très grand et très puissant. Tout ce que je pourrai en dire viendra de cette situation de responsabilité qui est religieuse en ce que le Moi ne peut pas l'éluder" (ibid., p. 110).

3 "Transcendance et Hauteur", repris dans Emmanuel Lévinas, Cahier de l'Herne, p. 109. Voir aussi Totalité et Infini, p. 188-189 et 256-257, ainsi que le texte suivant: "Entre les hommes est absente cette sphère du commun que toute synthèse présuppose. L'élément commun qui permet de parler d'une société objectivée, et par lequel l'homme ressemble aux choses et s'individualise comme une chose, n'est pas premier. La véritable subjectivité humaine est indiscemable, selon l'expression de Leibniz, et par conséquent ce n'est pas comme des individus d'un genre que les hommes sont ensemble" (Ethique et Infini, p. 82-83). Cfr. mon livre Transcendance et éthique. Essai sur Lévinas, Ousia 1989, chapitre 2. 
Or, pour penser cette situation qui saisit le propre de l'humain, les catégories de la logique du genre font défaut. Il faut reconnaître qu'ici s'établit une relation entre des termes différents et irréductibles, mais il ne faut pas oublier que ces termes - moi et autrui - sont séparés, ce qui veut dire qu'ils ne sont pas unis par la communauté du genre; et toutefois cette "séparation liante de la sociéte" - comme Lévinas l'a appelée une fois (Préface à S. Mosès, Système et révélation, p. 16) - noue une intrigue de sens où les notions mêmes d'intelligibilité et de relation entrent en jeu d'une manière nouvelle. L'ordre de l'humain, en tant qu'il est irréductible à la totalité, implique un pluralisme, une dimension du collectif et du social, où "la structure formelle de la relation" n'épuise pas "l'essence de la relation", ce qui "ne réaliserait pas le pluralisme" et reconstituerait la totalité (Totalité et Infini, p. 195). "Pour maintenir la multiplicité, il faut que la relation allant de moi à Autrui - attitude d'une personne à l'égard d'une autre - soit plus forte que la signification formelle de la conjoction où toute relation risque de se dégrader" (p. 93).

Or, c'est précisément la "religion" qui assure ce maintien de la multiplicité ou du pluralisme, parce qu'elle fait signe vers une situation ultime qui sous-tend la structure formelle et synthétique de toute relation, et la protège de sa dégradation en totalité. La signification concrète de la religion est donc un noeud paradoxal qui se noue entre des termes séparés, dont la proximité ou le rapport n'abolit pas la distance infranchissable, mais où en même temps cette distance n'atténue pas le caractère inévitable de la référence de l'un à l'autre, pourvu toutefois que cette référence ne soit pas comprise comme une référence logique ou psychologique - comme une référence que la réflexion pourrait découvrir - mais comme un événement éthique: l'événement d'une responsabilité pour l'autre qu'on ne peut pas éluder. C'est précisément la concrétude exceptionnelle de cette implication asymmétrique de l'un pour l'autre dans la relation humaine qui déformalise la notion de relation et permet de décrire phénoménologiquement la "mise en scène" de sa signification positive, en sauvegardant la séparation entre ses termes et leur proximité. La religion est la structure concrète et ultime du face-à-face, "relation sans relation" (p. 52), "rapport sans rapport" (p. 271), qui ne totalise pas les êtres séparés, qui ne les subordonne pas au savoir et à la thématisation, mais où cependant "l'un pèse ou importe ou est signifiant à l'autre" (En découvrant l'existence, p. 225), où ils sont liés par une "intrigue éthique antérieure à la connaissance" (Hors sujet, p. 236).

Dès lors, étant donné que ce rapport de moi à autrui - cette responsabilité primordiale et irréfutable - nous met en relation avec le tiers, et par conséquant se coule dans la forme du Nous (cfr. Totalité et Infini, p. 276), il faut penser ce Nous comme une pluralité qui ne soit pas "le pluriel de Je", ce qui veut dire que le social n'est pas une simple multiplication ou totalisation de l'individuel, mais une dimension originale qui "nous fait sortir des catégories d'unité et de multiplicité qui valent pour les choses, c'est-à-dire valent dans le monde d'un sujet isolé, d'un esprit seul" (De l'existence à l'existant, p. 163). Il faut insister sur la fonction de la religion - de la liaison irréfubatle de l'un à l'autre - dans cette pensée de la pluralité humaine irréductible à la totalité. Cela implique d'abord un refus du solipsisme, parce que seule une compréhension du subjectif ayant déjà en soi l'ouverture à l'extériorité - la liaison religieuse à l'altérité - est à même de saisir le caractère original de la pluralité humaine, son irréductibilité à une complication de solitudes juxtaposées et par là même totalisables. Par contre, le Nous pensé comme pluriel de Je est précisément une dimension d'intersubjectivité encore intérieure à une subjectivité isolée, dépourvue de l' extériorité 
d'autrui qui n'est jamais une extériorité simplement spatiale et numérique, mais une extériorité radicale, que Lévinas appelle pour cette raison "métaphysique". "Ainsi la relation métaphysique - dit-il - réalise-t-elle un exister multiple, le pluralisme", et il ajoute: "П faut expliciter le pouvoir qu'ont des êtres placés dans la relation de s'absoudre de la relation. Ce pouvoir comporte pour chacun des termes séparés, un sens différent d'absolution" (Totalité et Infini, p. 195).

C'est pourquoi Lévinas critique l'abolition du pluralisme que réalise la politique laissée à elle-même, "pluralité qui exprime la multiplicité des articulations d'un système, "monde sans multiplicité" où "les interlocuteurs renoncent à leur unicité" (p. 192). Le pluralisme de la différence, le différer même de la différence, le fait que chaque terme séparé s'absout différemment de la relation qu'il entretient et par là même est unique, assure à la fois la discrétion de la totalité et la relation à l'altérité. Cette prolifération de la différence dans l'intériorité de chaque terme de la relation sociale articule la résistance à la totalité, et interrompt "la tyrannie de l'universel et de l'impersonnel, ordre inhumain quoique distinct du brutal" (p. 219).

Lévinas est très attentif au glissement vers la tyrannie immanent à tout ordre étatique (cfr. p. 151), incapable de reconnaître l'individu "dans sa singularité" (p. 220). "La différence entre "apparaître dans l'histoire» (sans droit à la parole) et apparaître à autrui tout en assistant à sa propre apparition - distingue encore mon être politique de mon être religieux. Dans mon être religieux je suis en vérité (p. 231), car ce n'est que là que je suis reconnu dans ma singularité unique.

Je crois que c'est précisément par le biais de cette mise en garde contre le danger d'étatisme et de tyrannie immanent à toute politique et impliquant la négation de sa visée égalitaire - danger évidemment réalisé au degré extrême dans et par le totalitarisme - que la pensée de Lévinas fait signe vers la naissance de la vraie question politique. Celle-ci consiste dans la recherche d'un accord équitable entre volontés réellement séparées, uniques, c'est-à-dire irréductibles à la raison qui, à leur insu, les unirait et en constituerait l'en-soi provisoirement caché. Sans la multiplicité radicale de l'humain, sans l'irreductibilité du social à la totalité, l'"espace public" de la politique ne serait qu'une fiction, et la question qu'elle pose serait déjà résolue et par là même dissoute par la théorie philosophique, censée capable de saisir la raison universelle, unique et déjà donnée, dont les volontés particulières ne seraient que l'articulation contingente.

Cet enracinement du pluralisme dans l'íntériorité subjective "qui garantit la discrétion de la totalité" (p. 90), va de par chez Lévinas avec sa critique philosophique de l'idée de totalité, dont il rappelle qu'elle est venue en effet "après une expérience politique que nous n'avons pas encore oubliée (Ethique et Infini, p. 83). Allusion discrète au totalitarisme, dont la réflexion lévinassienne sur le politique et ses limites doit être lue comme une réplique, et à quoi il faut rattacher sa référence non théologique et non confessiornelle à la religion comme condition de l'égalité. "Nous proposons - écrit-il en effet - d'appeler religion le lien qui s'établit entre le Même et l'Autre, sans constituer une totalité" (Totalité et Infini, p. 10). Ce lien paradoxal constitue la situation ou la structure ultime à la base de toute synthèse formelle tendant à totaliser le Même et l'Autre, par exemple déjà dans le discours qui les nomme ensemble.

"Religion" dessine ainsi avant tout une conjoncture de transcendance, rompant la totalité et irréductible à une totalité, que Lévinas appelle également relation métaphysique ou relation éthique, et qui sauvegarde l'immédiateté absolue du face-à-face. "La conjoncture entre le Même et l'Autre où leur voisinage verbal déjà se tient, est l'ac- 
cueil de front et de face de l'Autre par moi. Conjoncture irréductible à la totalité, car la position de "vis-à-vis" n'est pas une modification de l'àlcôté-de...". Même quand j'aurai relié Autrui à Moi par la conjonction "et", Autrui continue à me faire face, à se révéler dans son visage. La religion soustend cette formelle totalité" (p. 53).

On peut voir là une confirmation de l'opposition lévinassienne à toute pensée du social en termes de totalité, opposition qui vise les philosophies de la communion, fascinées par l'idéal de la fusion, comprise toujours par Lévinas comme une sorte de juxtaposition extrinsèque qui s'ajoute à un solipsisme original. Il faut se référer ici à la première lecture de Heidegger par Lévinas dans les années trente et quarante, quand il s'éloigne déjà résolument de la "conception heideggerienne qui envisage la solitude au sein d'une relation préalable avec l'autre. [...] La préposition mit (avec) décrit ici la relation. C'est, ainsi, une association de côte à côte, autour de quelque chose, autour d'un terme commun, et, plus précisément pour Heidegger, autour de la vérité. Ce n'est pas la relation du face-à-face" (Le temps et l'autre, p. 18-19). Ce qui est paradoxal, c'est que pour Lévinas cette conception heideggerienne de la solitude, quoique ontologiquement dérivée en égard au rapport préalable et originaire avec l'autre, "loin de rompre avec la solitude, ne fait que la dilater". ${ }^{4}$ En effet, pour Heidegger le social ne se constitue pas autour de la relation immédiate avec autrui, mais autour d'un terme "qui sert d'intermédiaire, qui fournit le commun de la communion" (De l'existence à l'existant, p. 162), et qui par là même ne fait que confirmer le solipsisme. "Aussi comme dans toutes les philosophies de la communion, la socialité chez Heidegger se retrouve toute entière dans le sujet seul et c'est en terme de solitude que se poursuit l'analyse du Dasein, dans sa forme authentique. A cette collectivité de camarades, nous opposons la collectivité du moi-toi qui la précède" (ibid.). Cette denière n'est pas une communion, elle "dessine un pluralisme qui ne fusionne pas en unité" (Le temps et l'autre, p. 20), et par voie de conséquence permet de sortir de la solitude ontologique, précisément parce que la socialité est radicalement extérieure au sujet seul. Le rapport à autrui dans le face-à-face n'annule pas la séparation ni l'unicité de ses termes, mais d'autre part arrête le solipsisme d'une philosophie pour laquelle le Dasein ne comprend son être de façon authentique que dans l'exacte mesure où il existe à dessein de soi seul. ${ }^{5}$

Le rapprochement établi par Lévinas entre religion et désir, d'un côté, et politique et bonheur, de l'autre, suggère que le rapport asymétrique à autrui qui constitue à la fois la condition et la limite "religieuses" du politique - comme instauration de la réciprocité et de l'égalité - est d'ordre symbolique. Il s'agit d'un rapport qui ne saurait s'accomplir dans l'ordre du réel, car autrui se dérobe toujours, échappe à la réalité, s'y absout. Le désir de l'autre est irréductible à tout besoin qu'on pourrait satisfaire, précisément parce que l'autre ne se donne pas dans le réel. Toute la "phenoménologie" de l'approche d'autrui élaborée par Lévinas s'attache à cette impossibilité de remplir la visée subjective d'autrui, si bien que le désir, à la différence du besoin, se nourrit de sa propre faim. Si la satisfaction des besoins est le bonheur d'un être sensible, la perfection d'un être désirant est le caractère inassouvissable de son sésir.

Or, un tel désir - irréductible au bonheur assuré par la politique en tant que lutte pour l'instauration d'une société d'égaux, et assimilé à la religion - peut être considé-

4 J. Taminiaux, "La première réplique à l'ontologie fondamentale", Emmanuel Lévinas, Cahier de l'Heme, p. 284.

5 Je développe ce point dans "De l'errance à la responsabilité", Etudes phénoménologiques, n² 12, 1990. 
ré comme l'ouverture de l'humain à son propre, l'ouverture à la dimension de la pluralité, la sortie du solipsisme et par là à la fois la condition et la limite de la politique. En ce sens, la religion, comme lieu de cé "Désir d'Autrui qui est notre socialité même (En découvrant l'existence, p. 193), serait la figuration symbolique de l'extériorité de la société à elle-même, sans quoi la politique ne serait plus possible comme instauration d'une société d'égaux, mais aboutirait à la fausse communion de la totalité. En effet, on l'a déjà vu, "la politique laissée à elle-même, porte en elle une tyrannie. Elle déforme le moi et l'Autre qui l'ont suscitée, car elle les juge selon les règles universelles" (Totalité et Infini, p. 176).

Il faut donc penser la nécessité et les limites de l'ordre universel de la politique, où s'achève la lutte pour la reconnaissance, mais où l'égalité ne s'instaure pas si la singularité perd sa séparation et se dissout dans le collectif, ou dans la totalité. Je crois que la conception lévinassienne du religieux comme condition de l'instauration de l'égalité propre à la politique, peut donner à penser l'empiétement du caractère institué du politique et de son irréductibilité à une totalité, ainsi que l'impossibilité de les sauvegarder ensemble sans faire signe vers l'extériorité inassumable de la société à l'égard d'elle-même. Extériorité non spatiale mais d'ordre symbolique, quoique d'un symbolique conçu non pas comme un produit de l'activité subjective mais comme son lieu originel, comme lieu de la subjectivation et de la socialisation.

Tant de précisions et précautions sont ici indispensables, dans la mesure où il s'agit de parcourir le texte déjà classique de Lévinas pour y chercher une inspiration qui aide à penser des questions qui ne sont peut-être pas en tant que telles les questions qu'il a abordées et que son oeuvre a fait rebondir. En effet, on ne saurait négliger que Totalité et Infini a été au début de ce que Dominique Janicaud a magistralement analysé comme le tournant théologique de la phénoménologie française contemporaine, jusqu'à préconiser une sorte de confusion méthodologique entre les régistres du discours philosophique et les régistres du discours théologique. Ainsi ne peut-on pas oublier non plus que l'oeuvre de Lévinas a été parfois considérée comme une "manière nouvelle de parler de Dieu à l'homme contemporain", à l'âge de la mort de Dieu et de l'athéisme de masse. Mais indépendamment des intentions déclarées de l'auteur - toujours à vrai dire très nuancées - il est possible et légitime de s'interroger sur les effets de sens des aspects philosophiques de sa référence à la religion comme condition de l'égalité politique, pour y décéler une sorte de "mise en scène" d'une question théorique qui se situe peut-être au coeur d'une pensée radicale de l'institution de la démocratie. J'entends par là un space problématique qui ne coïncide pas avec la question de l'éclipse du religieux en Occident ou de son éventuelle résurgence sous telle forme ou telle autre, plus ou moins liée à un nouvel essor de l'ethos démocratique, qui d'autre part, quant à lui, est à vrai dire radicalement ménacé par la persistance et par la croissance de fondamentalismes religieux de toute sorte. Ce qui m'intéresse, ce n'est que la possibilité de retrouver dans la notion lévinassienne de religion comme condition de l'égalité politique en deçá ou au delà de la totalité, une figure de l'articulation entre la société et son extériorité, où, comme le suggère Claude Lefort, la philosophie peut voir préservée l'experience d'une différence qui n'est pas à la disposition des hommes, et qui les met en rapport avec leur humanité, laquelle s'ouvre à elle-meme étant prise dans une ouverture qu'elle ne fait pas (cfr. Essais sur le politique, p. 262-3).

L'empiétement déjà signalé entre l'institutionnel et le symbolique me paraît faire signe vers une impossibilité de penser l'oeuvre de la politique - l'instauration d'une 
société d'égaux, comme Lévinas le dit dans Totalité et Infini, ou la "comparaison des incomparables", comme il s'exprime dans Autrement qu'être (p. 199-205) - en termes d'immanence et de transparence absolues. La relation sociale implique l'établissement d'un pied d'égalité entre des individus séparés et autres: le propre de l'institution politique de la société est précisément cette égalisation - jamais absolue - des incomparables; qui ne se réalise pas sans présupposés, mais qui implique la reprise par la société diu caractère inassumable de son propre être. La société est déjà là, déjà donnée dans le gest même qui accomplit l'égalisation originaire des sujets sociaux: à l'origine de la société il y a donc déjà la société, non point comme une donnée naturelle ou sur-naturelle, mais comme la reprise instituante d'une donée déjà institué. Penser l'institution revient alors à penser son caractère originiare, l'imposibilité de sortir de l'articulation instituant/institué pour immaginer un point zéro de l'institution où l'instituant, au lieu d'être altération permanente d'un institué d'ores et déjà là, serait pure immanence à soi et production libre de l'institué.

L'altérité de l'instituant et de l'institué, comme altérité immanente à la societé qui se préssupose dans son être origine de soi, se double ainsi d'un rapport entre la societé et son extériorité à l'égard d'elle-même, extériorité symbolique qu'on ne saurait précipiter dans le réel - comme. c'est le cas dans les religions confessionnelles mais qui cependant introduit une articulation interne dans l'oeuvre même de l'institution. Articulation interne ou clivage, où dans le cercle originaire de l'institution, celleci est toujours la reprise de ce qui est déjà là. Nous sommes d'ores et déjà dans l'institué; l'instituant n'est qu'une altération incessante d'un institué toujours déjà là; l'institution primordiale, l'Urstiftung, est une notion-limite et mythique. L'activité de l'institution comporte ainsi une dimension inassumable et irréductible de passivité.

La référence lévinassienne au religieux comme condition du politique que j'essaye d'interroger ici - et qui n'épuise pas la signification de la religion dans l'oeuvre de Lévinas - décrit la relation à une alterité qui s'absout de la relation, qu'on ne peut donc pas précipiter dans le réel, qui se dérobe toujours à sa réification, et qui par là même met en scène une visée symbolique du social et l'inévitable écart de celle-ci par rapport à l'ordre du réel.

L'égalisation de ce qui est absolument autre, la comparaison des incomparables, en quoi consiste le propre de l'ordre social, à la fois sa prémisse et son résultat, implique donc la référence du social à une dimension symbolique qui garde son extériorité inassumable par rapport aux termes de la relation sociale. C'est dans ce sens que je lirais dans le dernier chapitre d'Autrement qu'être l'impossibilité de penser jusqu'au bout le social comme origine de lui-même et la nécessité d'introduire "le 'passage' de Dieu" pour penser "le retournement du sujet incomparable en membre de société (p. 202). Il s'agit, comme on sait, d'un point très délicat de la pensée de Lévinas, qui mériterait une analyse beaucoup plus détaillée. Dans l'économie de cette discussion, je me bornerai à suggérer que le recours à Dieu - qu'il faut entendre, à la lumière de l'article déjà cité sur "Le Moi e la totalité", comme "point fixe extérieur à la Société et d'où viendrait la Loi (Entre nous, p. 34) - fournit la figure de l'extériorité symbolique de la société à l'égard d'elle-même, à partir de quoi l'apparition originaire du tiers dans l'asymétrie du face-à-face se coule dans la forme du Nous sans se dégrader en "totalité impersonnelle", sans oublier la différence au profit de l'unité ou de la communion fusionelle. Dans ce sens l'universalité de la loi, la visibilité qu'elle instaure, institue l'égalité sans annuler "l'attention à Autrui en tant qu'unicité et visage (que le visi- 
ble du politique laisse invisible) et qui ne peut se produire que dans l'unicité d'un moi" (Totalité et Infini, p. 276-277).

La tyrannie de la politique laissée à elle-même consisterait alors dans la prétention de réduire l'ordre universel instauré par la loi à un ordre anonyme et neutral déraciné de son origine dans la relation sociale où proximité et distance vont de pair, où personne n'est à l'origine de l'institution, mais où entre les êtres séparés l'apparition du tiers pose l'exigence d'un plan commun, d'un pied d'égalité qui soit extérieur aux termes de la relation sans être à son tour un terme, sans être une projection de l'un d'eux, sans être objectivable ni réifié. Dans Autrement qu'être Lévinas introduit la référence à Dieu dans l'acte même "qui institue 'avec l'aide de Dieu', le lieu originel de la justice" (p. 204), à savoir l'ordre universel de la loi qui instaure l'égalité, mais qui demeure "au sein de la proximité" (p. 202) sans se confondre avec une généralité anonyme. Une telle universalité qui surgit de la proximité suppose "Dieu" comme "point fixe extérieur à la société". Qu'on lise ce passage difficile, mais typique du language at du raisonnement de l'ouvrage: "Dans la proximité l'autre m'obsède selon l'asymétrie absolue de la signification, de l'un pour l'autre [...]. La relation avec le tiers est une incessante correction de l'asymétrie de la proximité où le visage se dé-visage. Il y a pesée, pensée, objectivation et, par là, un arrêt où se trahit ma relation an-archique à l'illéité, mais où elle se traduit devant nous. Trahison de ma relation anarchique avec l'illéité, mais aussi une relation nouvelle avec elle: c'est grace à Dieu seulement que sujet incomparable à Autrui, je suis abordé en autre comme les autres, c'est-à-dire "pour moi". "Grâce à Dieu" je suis autrui pour les autres" (p.201).

La présence des guillemets qui toujours abritent dans ces pages la référence à l'aide" ou au "passage" ou à la "grâce" de Dieu ne doit pas être sous-estimée dans le texte d'un phénoménologue. Elle pourrait peut-être confirmer l'idée que la religion est ici en jeu non pas dans son sens théologique et confessionnel, mais pour suggérer l'implication nécessaire d'une dimension symbolique qui constitue la condition de l'institution politique de l'égalité. D'autre part, Lévinas reconnait que "Dieu n'est pas "en cause" comme un prétendu interlocuteur" (ibid.), et surtout qu'il s'agit d'un Dieu toujours renégable et en danger permanent de se muer en protecteur de tous les égoismes" (p. 205). Celà autorise, me semble-t-il, une lecture de cette référence à Dieu comme condition de l'égalité politique, qui souligne fortement l'impossibilité de la précipiter dans le réel, de la réduire à une origine mythique en deçà de la société dont celle-ci serait une conséquence. Lévinas parle d'un "en-deçá anarchique témoigné énigmatiquement, certes - dans la responsabilité pour les autres" (p. 203). Par là il fait allusion à l'anachronisme ${ }^{6}$ du pré-originel "supposé autrement qu'un principe n'est supposé par la conséquence dont il est synchrone" (ibid.). Dès lors, ce pré-originel dont on ne saurait déduire quoi que ce soit, signifie à partir de sa reprise dans l'ordre social où a lieu "l'apparition du tiers", qui est bien "l'origine même de l'apparoir, c'està-dire l'origine même de l'origine" (p. 204). L'implication immémoriale de la responsabilité de l'un pour l'autre - "affection an-archique" (p. 189) car antérieure à toute origine, anachronique - déformalise dans l'ordre social la référence au pré-originel, à l'Infini, à Dieu. Voilà la condition de l'égalité instaurée par la politique, son en-deçà, une dimension symbolique qui précède l'institution et lui échappe, sans en être l'origine, l'arché ou le principe, dimension symbolique d'où aucune institution n'est déductible, mais dont l'institution doit toujours être la reprise. Certes l'égalité est instaurée par

6 Je renvoie à mon texte sur "L'anacronismo", Postface à ma traduction d'Emannuel Lévinas - Adriaan Peperzak, Etica come filosofia prima, Milan 1989. 
l'institution de la loi politique, qui doit aussi la préserver et la maintenir: mais elle peut le faire en tant que reprise et visée d'un pré-originel qui chez elle continue de faire sens, qui en constitue la signifiance ultime, et qu'elle doit poser comme valeur. C'est cette dimension pré-originaire - qui n'appartient pas à l'ordre empirique du réel - que l'institution sociale doit viser, et qui lui permet d'instaurer l'égalité politique: une telle dimension est la mesure de sa légitimité, et de son irréductibilité à la totalité. 